Preprint typeset in JHEP style. - HYPER VERSION

\title{
The toes of the ultra high energy cosmic ray spectrum
}

\author{
Diego Harari ${ }^{a}$, Silvia Mollerach ${ }^{b}$ and Esteban Roulet $^{b}$ \\ ${ }^{a}$ Departamento de Física, FCEyN, Universidad de Buenos Aires \\ Ciudad Universitaria - Pab. 1, 1428, Buenos Aires, Argentina \\ ${ }^{b}$ Departamento de Física, Universidad Nacional de La Plata \\ CC67, 1900, La Plata, Argentina \\ Email: harari@df.uba.ar, mollerac@venus.fisica.unlp.edu.ar, \\ roulet@venus.fisica.unlp.edu.ar
}

\begin{abstract}
We study the effects of the galactic magnetic field on the ultra high energy cosmic ray propagation. We show that the deflections of the cosmic ray trajectories can have many important implications such as (de)magnification of the cosmic ray fluxes by lensing effects (which can modify the spectrum of individual sources), the formation of multiple images of a source or the existence of regions of the sky to which the Earth is almost blind. The appearance of image pairs is related to the existence of critical curves in the magnification maps, which divide regions in the sky where the images have opposite parities. The results are pictorially illustrated as the stretching and folding of a 'sheet' describing the sky seen on Earth. Making use of the most energetic AGASA events we emphasize the need to know the cosmic ray composition and the structure of the magnetic field when attempting to do detailed cosmic ray astronomy.
\end{abstract}

KEYWORDs: High-energy cosmic rays. 


\section{Contents}

1. Introduction 1

2. Cosmic ray propagation in the galactic magnetic field 2

2.1 Galactic magnetic field models 3

2.2 Cosmic ray deflections 6

3. Multiple images of cosmic ray sources 9

4. The observed spectrum 14

4.1 Energy-dependent flux amplification 14

4.2 Distortion of the spectrum of a point source 18

5. The Liouville theorem

6. Conclusions 20

\section{Introduction}

Ultra high energy cosmic rays (UHECRs) beyond the ankle of the spectrum, i.e. with energies above $5 \mathrm{EeV}\left(1 \mathrm{EeV}=10^{18} \mathrm{eV}\right)$, are most probably protons or nuclei of extra-galactic origin. The reason is that at those energies large rigidities would not allow their confinement by the galactic magnetic field and would also lead to excessively large anisotropies, added to the lack of plausible galactic sources for their acceleration to such enormous energies.

The major goal of existing and planned large air shower detectors is to unravel the nature and origin of these UHECRs, and one of the difficulties already encountered is the lack of obvious sources near the arrival directions of the highest energy events observed. Possible sources for these particles are AGNs, radio galaxies or $\gamma$ ray bursts. At the highest energies observed $(E \gtrsim 70 \mathrm{EeV})$ the attenuation of the fluxes by interactions with the cosmic microwave background radiation requires that the sources be not too far away, i.e. at less than $\sim 100 \mathrm{Mpc}$ in the case of protons [1] and even closer for heavier nuclei [2].

In their journey from their sources to the Earth, cosmic rays (CRs) are deflected by magnetic fields. A regular intergalactic field is strongly constrained $\left(B<10^{-9} \mathrm{G}\right)$ by rotation measures of extra-galactic radio sources. Galaxy clusters may have 
stronger magnetic fields, coherent over Mpc scales. It has been shown [3, [4] that a random component of strength $B \sim 0.1 \mu \mathrm{G}$ of the magnetic field of clusters (e.g. the Virgo cluster) can lead to a difussive propagation of protons up to energies as high as $100 \mathrm{EeV}$ as the cosmic rays exit or cross a cluster. Cluster magnetic fields can also lead to a change in the arrival direction of the UHECR and to energy dependent time delays, relevant for the detection of burst sources. They can also produce several images of a source [4]. The magnetic field of our Galaxy $(B \sim$ few $\mu \mathrm{G})$ can also lead to sizeable deflections, and the study of these deflections for different magnetic field models has been performed in refs. [5, 6], stressing the need to correct the observed arrival directions at Earth to obtain the direction of arrival outside the region of influence of the galactic magnetic field. The magnetic field of the solar system (few tens of $\mu \mathrm{G}$ ) has negligible effects on the deflection of the UHECR trajectories (although it may lead to some peculiar events when CR nuclei disintegrate when interacting with solar photons [7]).

In this work we study in detail the effects of the galactic magnetic field on the extra-galactic cosmic rays arriving to the Earth. We will determine the CR deflections in different realistic models for the magnetic field and for general cosmic ray composition (i.e. charge $Z$ ). Applying this to the highest energy $(E>40$ EeV) AGASA events [8] we illustrate the need to know the CR composition and to understand the details of the magnetic field when trying to locate the CR sources.

We then study the formation of multiple images of a source, something which turns out to be a quite common phenomenon. We also show that the CR deflections produce a magnetic lensing effect, which can sizeably amplify (or demagnify) the flux arriving from any given source. Since this effect is energy dependent, the energy spectrum seen on Earth will be different from that of the source.

We also show that the magnification of the CR flux by the galactic magnetic field becomes divergent for directions along critical curves in the sky seen from the Earth (corresponding to caustic curves in the 'source plane', i.e. in the corresponding directions outside the Galaxy). These caustics move with energy and as a caustic crosses a given source direction, pairs of additional images of the source appear or disappear. These and other features of the multiple images will be addressed in Sections 3 and 4 . As a final remark, we will discuss the relation of these results with the Liouville theorem, which implies that an isotropic flux outside the Galaxy (something that is not expected at UHEs) should remain isotropic on Earth.

\section{Cosmic ray propagation in the galactic magnetic field}

The trajectories of CRs arriving at Earth can be strongly affected by the galactic

magnetic field. A convenient way of computing the trajectory of a nucleus of charge $Z e$ and energy $E$ arriving at Earth from a given direction is to follow the trajectory of a particle with the same energy and opposite charge that leaves the Earth in 
that direction up to a point where the effect of the galactic magnetic field becomes negligible [9]. Cosmic rays of energy $E$ arriving from extra-galactic space in the direction in which the antiparticle left the galactic halo will arrive at Earth in the direction in which the antiparticle left the Earth. The antiparticle trajectories leaving the Earth can be obtained numerically solving the equation

$$
\frac{d^{2} \vec{x}}{d t^{2}}=-\frac{Z e c}{E} \frac{d \vec{x}}{d t} \times \vec{B}(\vec{x})
$$

using a realistic model of the galactic magnetic field.

\subsection{Galactic magnetic field models}

The Milky way, as well as other spiral galaxies, is known to have a large scale regular magnetic field. This is mainly determined by measurements of the rotation of the plane of polarization of radiation from pulsars and extra-galactic radio sources due to the Faraday effect. The rotation angle is proportional to the integral of the product of the electron density and the magnetic field component along the line of sight. Although there are still large uncertainties in these determinations and there is not a general agreement about the magnetic field structure, some characteristics are known [10]. The magnetic lines are thought to follow the spiral arms. The local value in the Sun's vicinity is $B \simeq 2 \mu \mathrm{G}$ and it points in the direction $\ell \simeq 80^{\circ}$.

Magnetic fields of spiral galaxies are distinguished between bisymmetric (BSS) and axisymmetric (ASS). BSS (ASS) fields are antisymmetric (symmetric) with respect to a $\pi$ rotation. In other words, in BSS models the field reverses its sign between the arms (assuming that there are two arms), while in ASS models it has the same orientation. A further classification of magnetic structures distinguishes fields that are symmetric $(\mathrm{S})$ or antisymmetric $(\mathrm{A})$ with respect to the Galaxy's mid-plane [10]. In the $\mathrm{S}$ models, the field has the same direction above and below the galactic plane, while in A models it is reversed ${ }^{1}$.

We use two combinations of the four classes of models to illustrate the effects of the galactic magnetic field on the propagation of CRs. We consider a bisymmetric model with even symmetry (BSS-S), which is the preferred model for our Galaxy, and some of the results are shown also for the axisymmetric model with odd symmetry (ASS-A), which somehow represents a possible extreme variation. We adopt a structure and strength very similar to those used by Stanev [5], but smoothed out in order to avoid spurious effects due to discontinuities in the field or its derivatives in the calculations.

In the galactic plane $(z=0)$ the field, directed along the spiral arms, has strength

$$
B_{s p}=B_{0}(\rho) \cos \left(\theta-\beta \ln \left(\rho / \xi_{0}\right)\right),
$$

\footnotetext{
${ }^{1}$ Note that in [5, 6] the $\mathrm{A}$ and $\mathrm{S}$ denominations are interchanged.
} 
for the BSS configuration [11. The angle $\theta$ is the azimuthal coordinate around the galactic center (clockwise as seen from the north galactic pole), $\rho$ is the galactocentric radial cylindrical coordinate, $\xi_{0}=10.55 \mathrm{kpc}$ corresponds to the galactocentric distance of the maximum of the field in our spiral arm and $\beta=1 / \tan p=-5.67$, where the pitch angle is taken as $p=-10^{\circ}$.

For the ASS model we adopt

$$
B_{s p}=B_{0}(\rho) \cos ^{2}\left(\theta-\beta \ln \left(\rho / \xi_{0}\right)\right) .
$$

The radial and azimuthal components in the galactic plane are given by

$$
\begin{aligned}
& B_{\rho}=B_{s p} \sin p, \\
& B_{\theta}=B_{s p} \cos p .
\end{aligned}
$$

We take

$$
B_{0}(\rho)=\frac{3 r_{0}}{\rho} \tanh ^{3}\left(\rho / \rho_{1}\right) \mu \mathrm{G},
$$

with $r_{0}=8.5 \mathrm{kpc}$ the Sun's distance to the galactic center and $\rho_{1}=2 \mathrm{kpc}$. This function has the $1 / \rho$ behavior at $\rho>4 \mathrm{kpc}$ as in [5] and [11] and goes smoothly to zero at the galactic center [11.

For the dependence on $z$, we consider a contribution coming from the disk and another from the halo. For the symmetric S models

$$
\vec{B}_{S}(\rho, \theta, z)=\vec{B}(\rho, \theta, z=0)\left(\frac{1}{2 \cosh \left(z / z_{1}\right)}+\frac{1}{2 \cosh \left(z / z_{2}\right)}\right)
$$

with $z_{1}=0.3 \mathrm{kpc}$ and $z_{2}=4 \mathrm{kpc}$. These scale heights are chosen so that this smoothed model gives a good fit to the model used by Stanev [5]. The field reversal at the galactic plane in the antisymmetric A models is taken into account adopting $B_{A}(\rho, \theta, z)=B_{S}(\rho, \theta, z) \tanh \left(z / z_{3}\right)$, with a negligible scale height taken as $z_{3}=$ 20 pc.

\subsection{Cosmic ray deflections}

The gyroradius of a $\mathrm{CR}$ nucleus with $E / Z=10 \mathrm{EeV}$ in a uniform magnetic field of strength $3 \mu \mathrm{G}$ is slightly larger than $3 \mathrm{kpc}$. The typical value of the large scale galactic magnetic field is a few $\mu \mathrm{G}$, and is approximately uniform over scales of the order of a few kpc. Thus the motion in the galactic magnetic field of nuclei with ratio $E / Z$ above $10 \mathrm{EeV}$ should be in general not very different from a quasirectilinear trajectory, with deflections away from the straight path smaller as larger is the $\mathrm{CR}$ energy. At lower energies the $\mathrm{CR}$ path lengths in the galactic magnetic field significantly increase with respect to the case of rectilinear propagation [12]. A turbulent component in the galactic magnetic field adds diffusion to the CR motion, but this effect is not expected to be significant at energies such that $E / Z \gtrsim 1 \mathrm{EeV}$ [13]. 
Figure 1 depicts trajectories of nuclei with $E / Z=1$ and $10 \mathrm{EeV}$ in the BSS-S galactic magnetic field model, that we will take as the reference one to illustrate most of the effects discussed in this work. When $E / Z=1 \mathrm{EeV}$ the nuclei follow helical trajectories that make a very large number of turns before their arrival to the Earth. These highly twisted paths are not exclusive of finely tuned, isolated incoming directions, but rather are a typical feature at these energies. Notice that the direction of entrance to the Galaxy need not be close to the disk in order for the UHECR to get trapped along the spiral structure of the magnetic field (dashed lines). When $E / Z=10 \mathrm{EeV}$ the CRs follow instead much straighter paths.

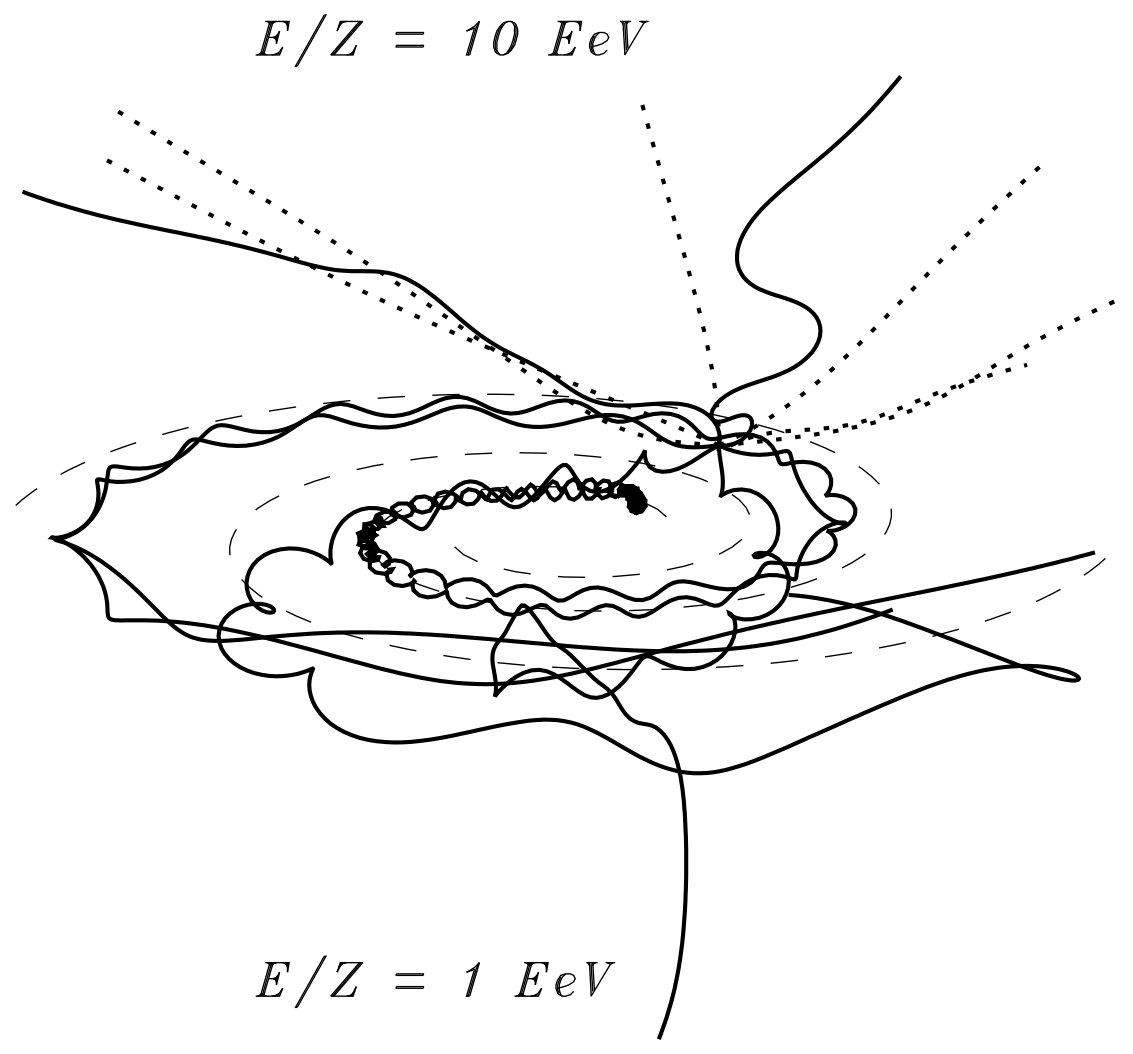

Figure 1: Examples of trajectories of nuclei with $E / Z=1 \mathrm{EeV}$ (solid lines) and $10 \mathrm{EeV}$ (dotted lines) in the BSS-S galactic magnetic field model. Particles reach the Earth at angles $\ell=30^{\circ}+n 60^{\circ}$, and $b=-20^{\circ}$ for $E / Z=1 \mathrm{EeV}$ and $b=20^{\circ}$ for $E / Z=10 \mathrm{EeV}$.

The transition between quasi-rectilinear and drift motion, in which CR trajectories turn around several times before they reach the Earth, occurs rather sharply at values below $E / Z \sim 3 \mathrm{EeV}$. Indeed, an average over a regular grid of arrival directions of the ratio between the distance traversed by a CR within $20 \mathrm{kpc}$ of the galactic center and the distance it would have traversed along a straight path gives a mean value of $1.01,1.14$ and 2.2 for ratios $E / Z=10,3$ and $1 \mathrm{EeV}$ respectively (in 
the reference BSS-S magnetic field model). The rms dispersions are 0.01, 0.18 and 1.7 respectively. Let us note that most of the excess corresponds to the increased path length within $\sim \pm 1 \mathrm{kpc}$ of the galactic plane.

Figure 2 illustrates how much do the arrival directions of charged UHECRs deviate from their incoming direction at the galactic halo due to deflections in the magnetic field.


Figure 2: Deflections of CR nuclei in the galactic magnetic field. Each diamond is an arrival direction at Earth. The points along each line as we move away from the diamond are actual incoming directions at the halo for decreasing CR energies. The dots correspond to $E / Z=100,30$ and $10 \mathrm{EeV}$ respectively. The tip of each arrow corresponds to $E / Z=$ $7 \mathrm{EeV}$. Top panel: BSS-S magnetic field configuration. Bottom panel: ASS-A model.

The plot is in galactic coordinates $\ell$ (longitude) and $b$ (latitude). The supergalactic plane (solid line) and the Earth equatorial plane (dashed line) are superimposed for visual guidance. A regular grid of UHECR arrival directions at Earth is 
mapped into the directions from which CRs of different energies entered the halo. In practice, the trajectories are followed up to distances $20 \mathrm{kpc}$ away from the galactic center, where the effects of the magnetic field in the models considered here are already negligible. The diamond at the origin of each line denotes the observed arrival direction. The points along the line from each diamond towards the tip of the respective arrow indicate the actual direction from which the CR entered the galactic halo for decreasing CR energy. The dots along each line correspond to nuclei with ratio $E / Z=100,30$ and $10 \mathrm{EeV}$ respectively. The tip of the arrow corresponds to the incoming direction at the halo of a $\mathrm{CR}$ with $E / Z=7 \mathrm{EeV}$. The top panel displays the result for the BSS-S galactic magnetic field model and the bottom panel corresponds to the ASS-A configuration.

This figure is the analogue of Figure 2 in Stanev's work [5] ${ }^{2}$. Deflections in the ASS-A model are symmetric with respect to the galactic plane, since $\vec{B}(z)=$ $-\vec{B}(-z)$. Deflections in the BSS-S case are neither symmetric nor antisymmetric with respect to the galactic plane, although there is an approximate antisymmetry at relatively high energies. In the ASS-A model there is a flow of lines out from the galactic plane. CRs from extra-galactic sources at relatively high latitudes will be observed at arrival directions closer to the galactic plane. From the opposite viewpoint, this flow of lines out from the galactic plane suggests that CRs from extra-galactic sources that lie very close to the galactic plane may be practically unable to reach the Earth below certain energies. This will be discussed in the next Section.

Notice also in Figure 2 that there are directions in the sky where the tips of the arrows converge, or even where different lines intersect. This indicates the possibility of observing, for some range of energies, multiple images of a single CR source, since one incoming direction at the halo is mapped into two or more arrival directions at the Earth. This possibility will also be discussed at length in the next Section. There are other peculiar features in Figure 2, such as sudden changes in the direction of some arrows. The source of this behavior will also become apparent from the discussion in the next Section.

As a concrete illustration of the impact that deflections in the galactic magnetic field may have upon UHECR observations, we plot in Figure 3 the arrival directions of all the events recorded by AGASA [8] with energies higher than $40 \mathrm{EeV}$ and the corresponding incoming direction at the galactic halo, depending upon the assumed CR composition. On top are the 17 AGASA events with energies higher than 60 $\mathrm{EeV}$, and at the bottom are the 30 events with energies between 40 and $60 \mathrm{EeV}$ (plus one event with energy just below $40 \mathrm{EeV}$, also included in Table 2 in 8 ). The diamonds denote the observed arrival direction of each event. The points along each line denote the actual incoming direction at the halo for nuclei with increasing $Z$ as

\footnotetext{
${ }^{2}$ Note that in 5] $\ell$ actually grows from left to right.
} 
we move away from each diamond towards the tip of the arrow. In the top panel the dots along each line indicate the results for protons $(Z=1)$, Carbon $(Z=6)$, Neon $(Z=10)$, Silicon $(Z=14)$, Calcium $(Z=20)$, and the tip of each arrow corresponds to Iron $(Z=26)$. In the case of the lower energy events $Z$ ranges from 1 to 10 . The deflections shown correspond to the BSS-S magnetic field configuration.


Figure 3: Observed arrival directions (diamonds) of AGASA events with energies larger than $60 \mathrm{EeV}$ (top panel) and energies between $40 \mathrm{EeV}$ and $60 \mathrm{EeV}$ (bottom panel), and the corresponding incoming directions of CRs at the galactic halo for increasing $Z$ as we move away from each diamond. The dots indicate the results for $Z=1,6,10,14$ and 20 and the tip of the arrow is for $Z=26$ (Iron) in the top figure. In the bottom figure $Z$ varies between 1 and 10 only. Deflections correspond to the BSS-S model.

Correlation between the observed events and known sources for a heavy (large $Z$ ) component of the UHECRs is clearly impossible without a detailed knowledge 
of the galactic magnetic field structure. Besides, clustering of observed arrival directions may not reflect clustering of the incoming directions at the galactic halo. The opposite is also a possibility: several quite separated events may arrive from the same or nearby incoming directions at the halo for particular values of $Z$.

\section{Multiple images of cosmic ray sources}

A very interesting and quite common effect resulting from the magnetic deflections is the appearance of multiple images of an extra-galactic UHECR source. This means that CRs arriving to the galactic halo from a given direction can reach the Earth following several different trajectories and are hence observed with different arrival directions. This can clearly be appreciated in Figures 4 and 5 where we have plotted for a regular grid of arrival directions at Earth in galactic coordinates $b$ and $\ell$, the direction from which the particles arrived to the galactic halo.

We move along 'horizontal' or 'vertical' lines following arrival directions at Earth with fixed $b$ or $\ell$ respectively. As the trajectories depend on the $E / Z$ ratio, the surface is stretched and folded as $E / Z$ changes. The three panels in Figure 4 show the development of the folding for the BSS-S model at energies $E / Z=30,10$ and $5 \mathrm{EeV}$. For large $E / Z$, the sheet is quite smooth as the effect of the magnetic field on the $\mathrm{CR}$ trajectories becomes small. For decreasing $E / Z$, foldings on the surface appear. CRs arriving from a direction in the halo where a folding has developed are seen at Earth from all the points of the grid that overlap in that place. For a source located in a direction in which a folding develops at small energies, we observe that the high energy apparent position of the source moves gradually for decreasing energies and at the energy at which the folding reaches the source direction, a pair of new images of the source appears in a different region of the sky. Alternatively, if a folding moves out of the source direction, a pair of images merges and disappears (if the sheet were to unfold just along the direction of the source, the three images would merge together into a single one, which is the magnetic analogue of crossing a caustic through a cusp in gravitational lensing theory). Notice for example that if a region which is folded in one direction (leading to a piece of sky with three images) develops another folding in a transverse direction as the energy decreases, six new images of a source located there will appear (leading then to a total of nine images for that source). Since it is apparent from the Figures that large areas of the sky develop foldings, multiple images should be a pretty common phenomenon. The fact that new images appear in pairs is a known fact in gravitational lensing theory [14].

Another interesting fact that can be appreciated in Figures 4 and 5 regards

the parity of the images observed. When a folding in the surface develops and a couple of new images of some source appears, one of the new images is a mirror reflected image of the source. In general every folding separates regions of images with opposite parity. 

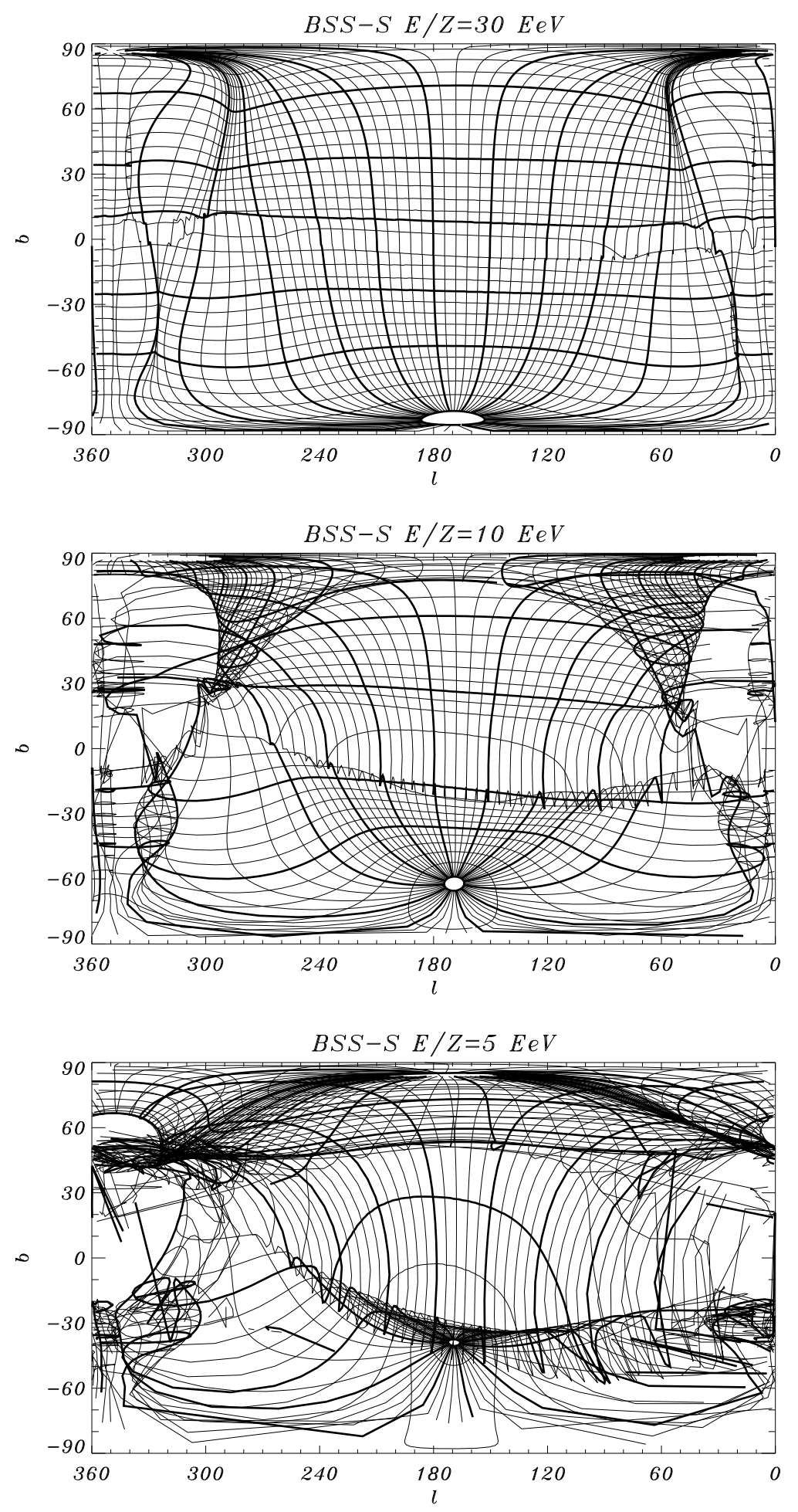

Figure 4: "Sky sheets": directions of incoming CRs in the halo that correspond to a regular grid of arrival directions at Earth, for the BSS-S magnetic field configuration with $E / Z=30$ (top), 10 (middle) and $5 \mathrm{EeV}$ (bottom). Sources located in regions where the sheet is folded have multiple images. 


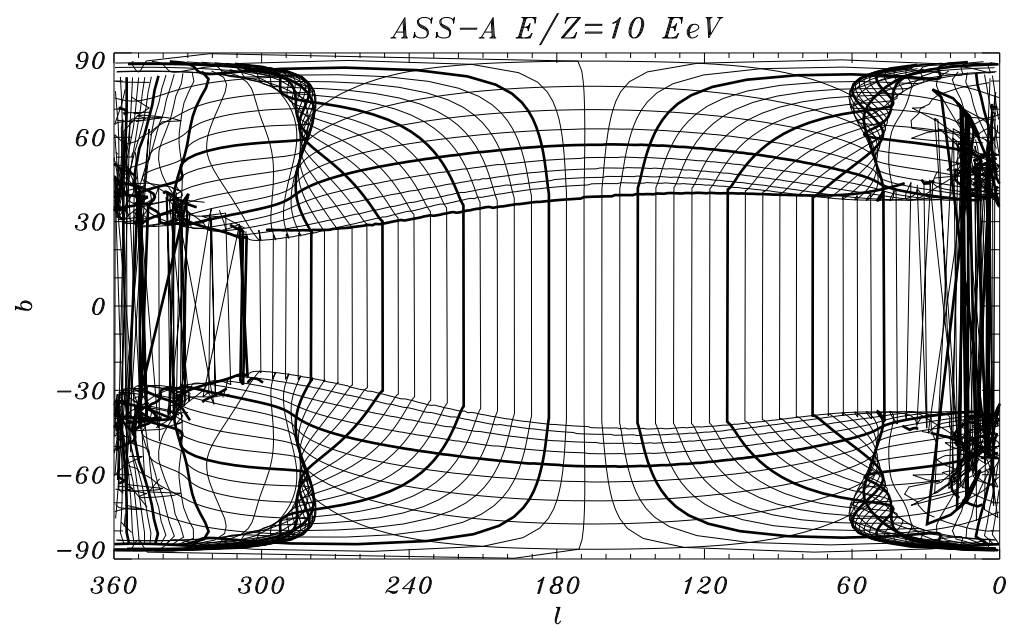

Figure 5: Same as Fig. 4 for the ASS-A magnetic field configuration and for $E / Z=$ $10 \mathrm{EeV}$ only. The Earth is practically blind to CR sources located in the very stretched region around the galactic plane.

Another curious effect that manifests in Figures 4 and 5 is the appearance of regions where the sheet is very stretched, and hence the incident fluxes will appear very demagnified. This means that $\mathrm{CR}$ sources located in those regions will become almost undetectable from the Earth. This is a rare effect in symmetric S models, associated essentially to regions near the galactic center. However, for the antisymmetric A models it is a very common effect, as the sky sheet presents a highly demagnified region all along the galactic plane, as shown in Figure 5. The reason is that antiparticles leaving the Earth with small angles above or below the galactic plane are deflected away from the plane. These deflections increase with decreasing energies and are already larger than $30^{\circ}$ for $E / Z=10 \mathrm{EeV}$ if $|b|>0.1^{\circ}$ in the Earth. Thus, for the sources located in a large area of the sky around the galactic plane $\left(|b|<30^{\circ}\right)$, the CRs can reach the Earth only if they come along a fine-tuned path which arrives very close to the galactic equator. We note that if we were to take $z_{3} \rightarrow 0$ (so that the change in sign of $B$ across the galactic plane in the A models were discontinuous), there would be no paths reaching the Earth from sources within $\pm 30^{\circ}$ in Figure 5. Hence, for all practical purposes it would be as if the "sky sheet" were teared up and divided into two disconnected pieces. These kind of tears appear when there is a discontinuity, i.e. when infinitesimally nearby trajectories leaving the Earth lead to widely separated directions in the halo ${ }^{3}$. In the $\mathrm{S}$ models (Figure 4), something qualitatively similar happens near the galactic center, and will lead to the "loss" of images in highly demagnified regions (see below).

From the data used to construct the grids in Figure 4 one can read the observed

\footnotetext{
${ }^{3}$ This is also the reason why in microlensing only two images exist when a point-like lens is considered. The third one falls in a tear with zero magnification.
} 
angular position(s) at a given energy of the image(s) of a source that injects CRs at a fixed direction in the halo. One can then follow the displacement in the observed angular position of each image as the CR energy is gradually changed, exploring at each energy step a neighborhood of the arrival direction from the previous step, and backtracking it to the halo until it reaches the appropriate injection direction with the required accuracy. One should start this process at low energies and then move up, otherwise one would only follow the evolution of the principal image of the source but miss the secondary images.
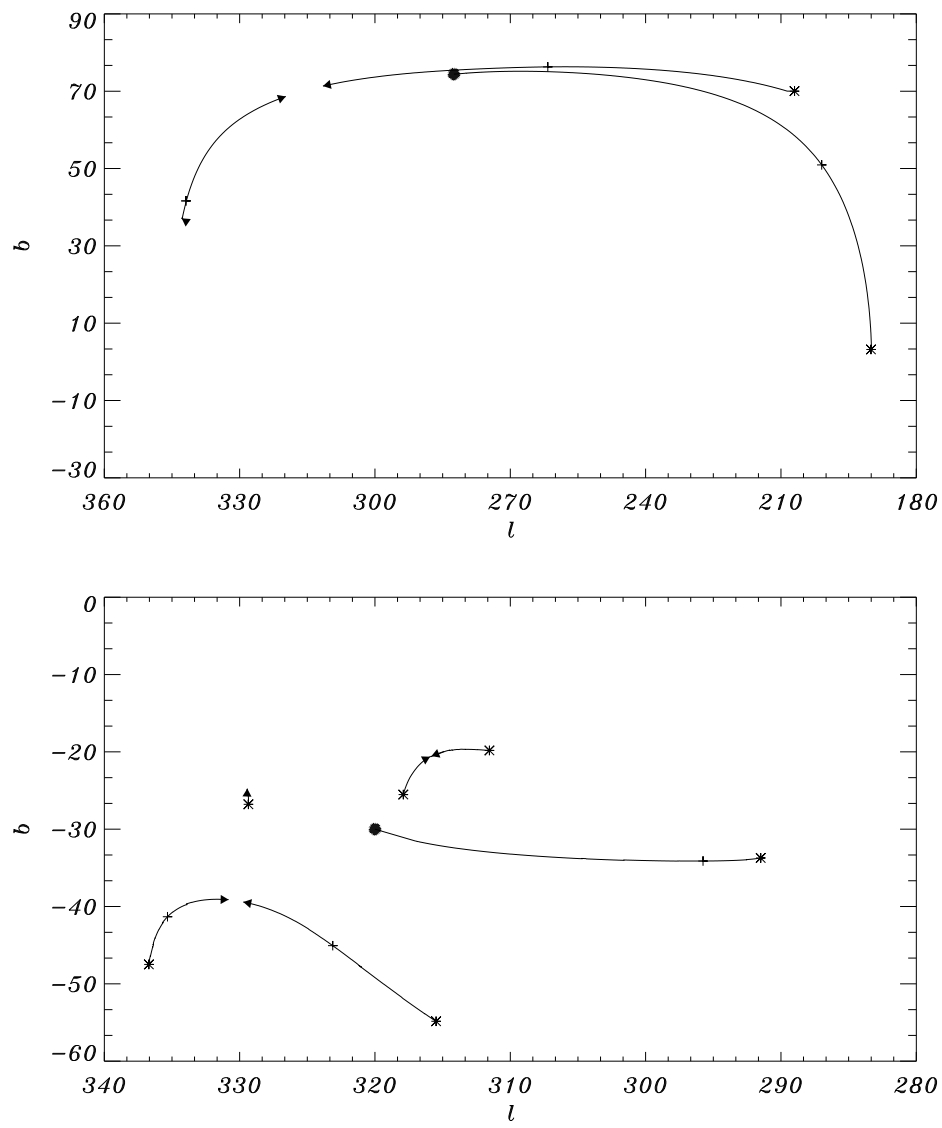

Figure 6: Illustration of the angular displacement of a source image as a function of energy, and of the formation and disapearance of secondary images. The actual source in the top panel is at $(\ell, b)=\left(284^{\circ}, 75^{\circ}\right)(\mathrm{M} 87)$ and the source in the bottom panel is at $(\ell, b)=\left(320^{\circ},-30^{\circ}\right)$. The magnetic field is taken in the BSS-S configuration.

Some examples of the effects discussed are illustrated in Figure 6 for two particular source directions, both lying near the super-galactic plane, adopting the BSS-S magnetic field configuration. The top panel corresponds to M87 in the Virgo Cluster (galactic coordinates $\left.(\ell, b)=\left(284^{\circ}, 75^{\circ}\right)\right)$. The dot is the actual source direction, which is the observed one at very high energies. As the $E / Z$ ratio decreases, the apparent position moves along the line. When $E / Z$ reaches around $20 \mathrm{EeV}$, a pair 
of new images are created at the point where two arrows meet, and the images then move along the lines shown, one to the right and the other to the left. The plus signs indicate the position of the images at $E / Z=10 \mathrm{EeV}$. Just below this value the image on the left falls into a region of negligible magnification near the galactic center and hence "disappears", in the sense that the path leading to it is so fine-tuned that we were unable to find it. The asterisks show the locations of the two surviving images at $E / Z=5 \mathrm{EeV}$. Note that the images become separated by more than $150^{\circ}$ from each other.

The bottom panel in Figure 6 displays an example in the southern hemisphere, for a direction at $(\ell, b)=\left(320^{\circ},-30^{\circ}\right)$. Here two images are created at $E / Z \simeq 15 \mathrm{EeV}$ and another couple at $E / Z \simeq 6 \mathrm{EeV}$. There is also an additional image which appears just above $E / Z \simeq 5 \mathrm{EeV}$ (its companion image is very demagnified and we couldn't find it). Three images are then observed at $E / Z \simeq 10 \mathrm{EeV}$ (plus signs) and a total of six are 'visible' at $E / Z \simeq 5 \mathrm{EeV}$ (asterisks) ${ }^{4}$.

Finally, let us note that in gravitational lensing theory, the formation of multiple images can also be studied using Fermat's principle, i.e. looking for the paths between the source and the observer for which the light travel time is an extremum. Similarly, each CR trajectory arriving to the Earth is an extremum of the action for a charged particle in the galactic magnetic field, i.e. of

$$
S=\int_{\text {Source }}^{\text {Earth }} \mathrm{d} t \mathcal{L}
$$

where the Lagrangian is

$$
\mathcal{L}=-m c^{2} \sqrt{1-(v / c)^{2}}+\frac{q}{c} \vec{v} \cdot \vec{A}
$$

with $\vec{A}$ the magnetic field vector potential. The different extrema of the action $(\delta S=0)$ will give rise to the different paths along which the CRs can arrive to the Earth, and hence lead to the different images of the source.

We note that, since $|\vec{v}|$ is constant along the real trajectories, the kinetic piece of the action is just proportional to the path length traversed. At very high energies, $E / Z>100 \mathrm{EeV}$, only the kinetic term is relevant in the functional variation of the action, and hence a single image will be seen, corresponding to the almost straight propagation. This path is a minimum of the action.

For lower energies, the magnetic contribution becomes relevant and competes with the kinetic piece to produce new extrema, which can in principle be saddle points (in the sense that there will be (not infinitesimally) neighboring paths for which the action will increase, and others for which it will decrease) or even maxima.

\footnotetext{
${ }^{4}$ It is remarkable that these kind of 'footprints' are found in the sky when looking beyond the ankle of the CR spectrum.
} 


\section{The observed spectrum}

\subsection{Energy-dependent flux amplification}

As we mentioned before, the galactic magnetic field can act as a giant lens that amplifies or demagnifies extra-galactic sources. The magnitude of this effect depends on the direction of observation and on the $E / Z$ ratio. Consequently, the observed spectrum of a source can be affected, as the CR flux will suffer different amplifications for different energies. In the case of multiple imaging of a source, each of the images will be magnified by a different amount by the magnetic field.

This can be studied quantitatively by considering the focusing effect of the galactic magnetic field on a bundle of particles arriving from outside the Galaxy. When a bundle of charged particles with parallel trajectories arrives to the galactic halo and, after being deflected by the galactic magnetic field, reaches the Earth, it will be generally more focused or defocused. The amplification is given by the ratio of the flux of particles reaching the Earth to that outside the galactic halo. We compute the magnification of a source observed at Earth in a given direction and for a given $E / Z$ ratio by first backtracking an antiparticle leaving in that direction up to a distance of $20 \mathrm{kpc}$ from the galactic center, where the effect of the magnetic field becomes negligible. Then, we track the particle back to the Earth by reversing the final velocity of the antiparticle at the halo border. At the same time, we track also the position of two more particles entering the halo with trajectories parallel to the fiducial one, the same $E / Z$ value, but displaced in two orthogonal directions. We follow the evolution of the displacement of these two particles from the fiducial trajectory by solving the equations

$$
\frac{d^{2} \Delta \vec{x}_{i}}{d t^{2}}=\frac{Z e c}{E}\left(\frac{d \vec{x}_{0}}{d t} \times \Delta \vec{B}_{i}\left(\vec{x}_{0}\right)+\frac{d \Delta \vec{x}_{i}}{d t} \times \vec{B}\left(\vec{x}_{0}\right)\right),
$$

coupled to eq. (2.1). In the previous equation, $\vec{x}_{0}$ denotes the position of the fiducial particle, $\Delta \vec{x}_{i}(i=1,2)$ the displacements of the other two particles, and $\Delta \vec{B}_{i}\left(\vec{x}_{0}\right) \equiv$

$\vec{B}\left(\vec{x}_{0}+\Delta \vec{x}_{i}\right)-\left.\vec{B}\left(\vec{x}_{0}\right) \simeq\left(\Delta \vec{x}_{i} \cdot \nabla\right) \vec{B}\right|_{x=x_{0}}$. Note that in eq. (4.1) we have neglected a term proportional to $\Delta \vec{x}_{i} \times \Delta \vec{B}_{i}$ as it is of second order in small displacements. When the three particles arrive back to the Earth we compute the area subtended by the two displacement vectors as $A_{E}=\left.\left(\Delta \vec{x}_{1} \times \Delta \vec{x}_{2}\right) \cdot \hat{v}\right|_{E}$, obtaining the magnification associated to that direction as the ratio of the original area subtended at the halo border, $A_{H}$, and $A_{E}$.

We note that the linearity of eq. (4.1) in $\Delta \vec{x}$ implies that, if $\Delta \vec{x}(t)$ is a solution, so is $\alpha \Delta \vec{x}(t)$, with $\alpha$ a constant. This implies that all the points inside the initial rectangular area $A_{H}$ remain inside a parallelogram as they propagate, and arrive inside the $A_{E}$ defined above.

A dimensional analysis may be helpful to estimate under which conditions large (de)magnifications may be attained in a magnetic field. Assume that the conditions 
are such that the deflection of the fiducial trajectory in eq. (4.1) is small. Take then $\vec{x}_{0} \simeq s \hat{s}$, with $\hat{s}$ the unit vector along the initial direction of the fiducial trajectory and $s$ the distance from the entrance point at the halo border. The deflection angle is thus given by $\delta \simeq(Z e / E)\left|\int_{0}^{L} \vec{B}_{\perp}(s) d s\right| \simeq 5^{\circ} Z(10 \mathrm{EeV} / E)\left|\int_{0}^{L} \vec{B}_{\perp}(s) d s\right| /(1 \mu \mathrm{G} 1 \mathrm{kpc})$. Here $\vec{B}_{\perp}$ is the component of $\vec{B}$ perpendicular to the trajectory, and $L$ is the distance traversed by the particle in the magnetic field. Assume also that the relative deflections of two neighboring trajectories initially parallel to the fiducial one but displaced in orthogonal directions are also small, which implies small (de)magnifications. To the lowest order of approximation, the rhs of eq. (4.1) can be evaluated along undeflected trajectories, and the rate of change of the area $A$ subtended by the two displacement vectors is then given by

$$
\frac{d^{2} A}{d s^{2}} \simeq-\frac{Z e}{E} \hat{s} \cdot(\nabla \times \vec{B}) A_{0},
$$

where $A_{0}$ is the area initially subtended (and the initial condition $\mathrm{d} \Delta \vec{x}_{i} / \mathrm{d} t=0$ was used). After a distance $L$ the area becomes $A \simeq(1-2 \kappa) A_{0}$ where $\kappa=$ $(Z e / 2 E) \int_{0}^{L} d s(L-s) \hat{s} \cdot(\nabla \times \vec{B}){ }^{5}$ The magnification is $\mu=A_{0} / A \simeq 1+2 \kappa$. We stress the fact that this conclusion applies only to the case when both the deflection of the central trajectory is small and $\kappa \ll 1$. Besides, changes in the shape of the region subtended by the neighboring trajectories (its potential distortion from a rectangle into an arbitrary parallelogram) have also been neglected. In the language of gravitational lensing, this result applies when both (de)magnification and shear are small.

Even though the previous analysis applies to the case of small (de)magnification only, it serves its purpose to crudely estimate the conditions necessary to attain large focusing, which require $\kappa$ to be of order unity. Take, as a simple illustrative example, an approximately straight trajectory perpendicular to the galactic plane and incident from the north. In this case, $\kappa \simeq-0.9\left(Z e / 2 E r_{0}\right) \beta B_{0}\left(r_{0}\right) \sin \left(\beta \ln \left(r_{0} / \xi_{0}\right)\right)\left(z_{1}^{2}+\right.$ $\left.z_{2}^{2}\right) \simeq 0.13(100 \mathrm{EeV} Z / E)$, in the BSS-S model ${ }^{6}$. The corresponding deflection for the same incident trajectory is $\delta \simeq 0.8(Z e / E) B_{0}\left(r_{0}\right) \cos \left(\beta \ln \left(r_{0} / \xi_{0}\right)\right)\left(z_{1}+z_{2}\right) \simeq$ $2^{\circ}(100 \mathrm{EeV} Z / E)$, and hence sizeable magnifications do not require necessarily large deflections. For instance, we shall see that there are already divergent magnifications at $E / Z \simeq 30 \mathrm{EeV}$, even along directions for which deflections are not larger than $10^{\circ}$. Viceversa, a strong but almost constant field can produce large deflections without significant magnifications.

\footnotetext{
${ }^{5}$ The appearance of the factor $(L-s)$ here has a simple physical interpretation: the farther out that the lensing effect induced by $\hat{s} \cdot \nabla \times \vec{B}$ takes place, the more 'leverage arm' it has to focus the beam into a smaller area.

${ }^{6}$ Note that $\kappa$ will reverse sign for a CR entering from the south along the vertical. Note also that for directions near the poles and small magnifications, $\kappa \propto\left(z_{1}^{2}+z_{2}^{2}\right)$, and hence the halo field gives the dominant contribution around the poles.
} 

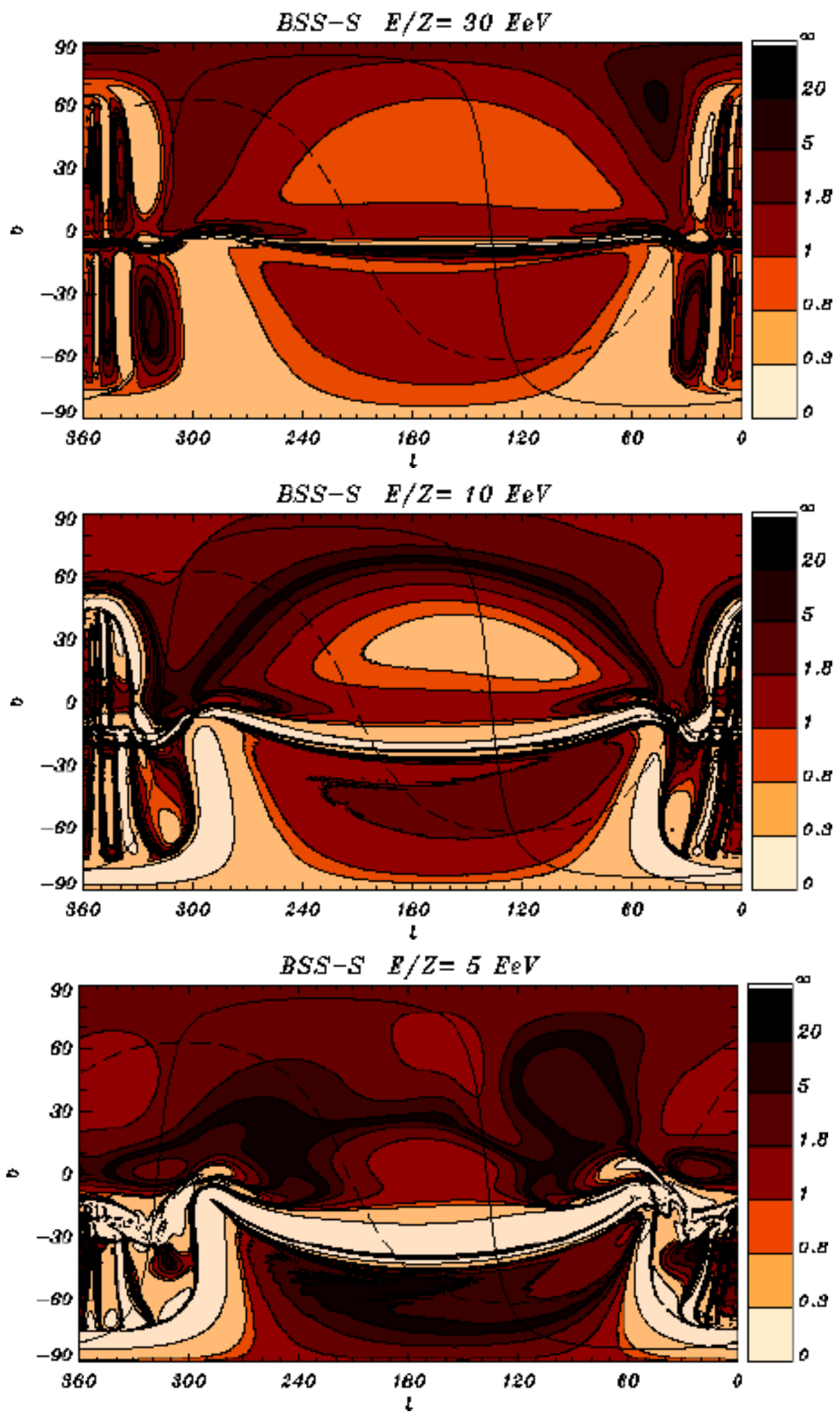

Figure 7: Contour plots of the magnification of the CR flux from a point source as a function of the arrival direction at Earth, in the BSS-S model and for $E / Z=30$ (top), 10 (middle) and $5 \mathrm{EeV}$ (bottom). 


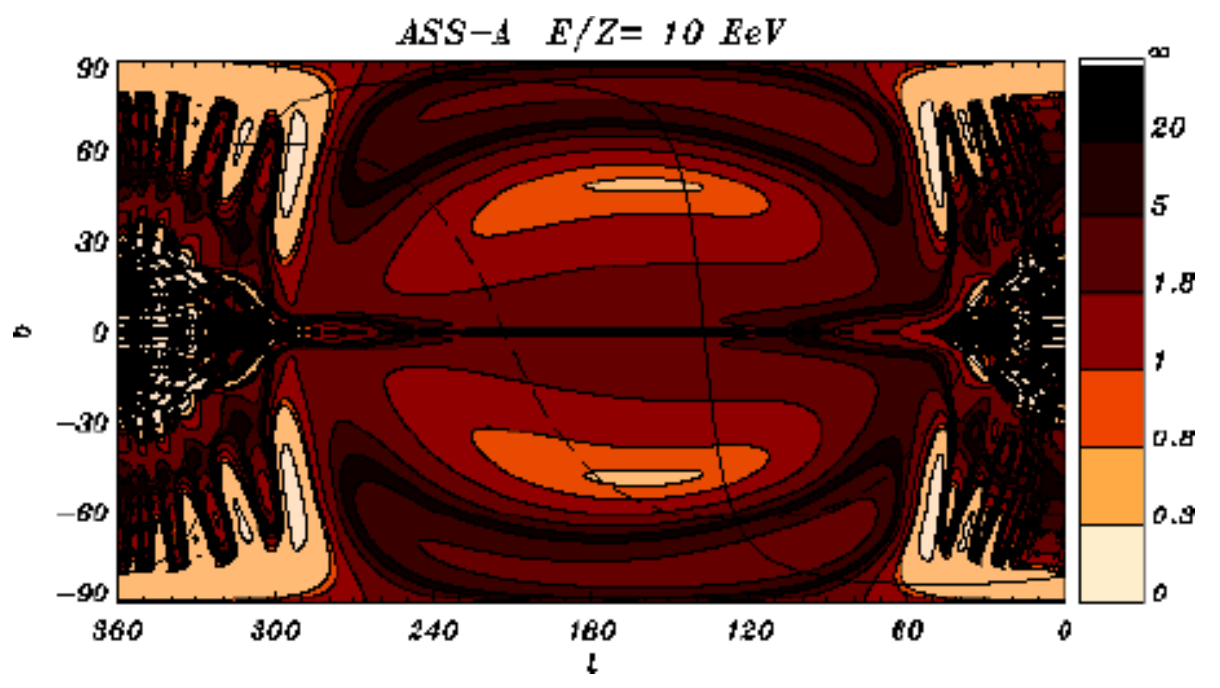

Figure 8: Same as Figure 7, now in the ASS-A model and for $E / Z=10 \mathrm{EeV}$.

Larger values of $\kappa$ than that estimated for the vertical direction may be expected for other directions along which more intense field gradients are encountered or longer distances are traversed within significant field gradients. Large (de)magnifications of UHECRs by the galactic magnetic field may thus occur when the ratio $E / Z$ is around and below a few tens of EeV, and even for larger values of this ratio in some special directions.

The precise determination of the amplification in the galactic magnetic field requires the numerical integration of eq. (4.1) coupled to eq. (2.1). We now turn to the numerical results of this calculation, computed as described around eq. (4.1). Figure 7 shows contour plots of the magnification as a function of the observed arrival direction at Earth in galactic coordinates, for the BSS-S magnetic field model and for ratios $E / Z=30,10$ and $5 \mathrm{EeV}$. Figure 8 is for the ASS-A model, at $E / Z=10 \mathrm{EeV}$. They were obtained through a $450 \times 900$ grid which covered regularly all directions in the observer's plane.

The regions of very large amplification reveal the existence of critical curves at which the magnification formally diverges (something which does never happen for realistic extended sources). It is instructive to compare these amplification contour plots with the "sky sheets" shown in Figures 4 and 5. For instance, the critical curves in the amplification maps at the observer's plane correspond to the caustics in the source plane (the foldings in the sky sheets). Notice also that since the foldings in the sky sheet separate regions of opposite image parity, the critical curves in the magnification maps must be closed curves, as is apparent in Figures 7 and 8.

The value of the magnification at a given direction in the maps of Figures 7 and 8 is the enhancement factor of the flux that arrives to the Earth in that direction from a point source. Since the magnification is a function of energy (for fixed $Z$ ) this effect changes the spectral slope of the UHECR flux from point sources. 
The ASS-A model produces a magnification map symmetric with respect to the galactic plane due to the odd nature of the field with respect to the $z$-coordinate. An approximate antisymmetry in the BSS-S magnification maps is apparent at the largest value of the $E / Z$ ratio. At lower energies this antisymmetry becomes less pronounced. It is quite evident that away from the galactic center, the regions in the northern galactic hemisphere tend to be magnified while those in the southern hemisphere tend to have significant demagnifications. This can make sources with the same injected spectrum have a harder observed spectrum in the South than in the North (i.e. steeper in the North).

\subsection{Distortion of the spectrum of a point source}

We now illustrate through some particular examples the dramatic changes upon the spectrum injected by a point source that arise due to the combined effect of flux (de)magnification and multiple image formation in the galactic magnetic field.
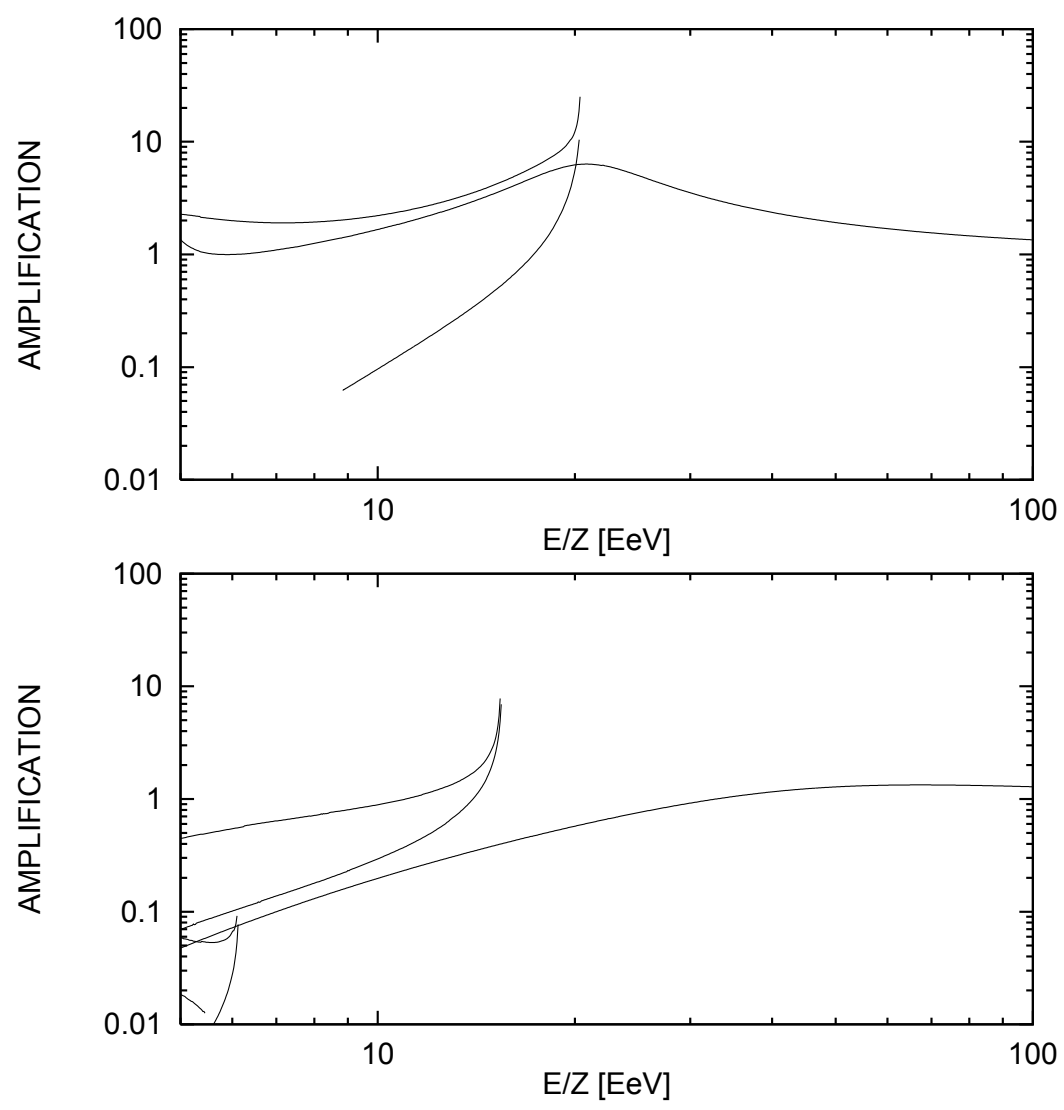

Figure 9: Energy dependence of the amplification factor of the principal and secondary images of a source at $(\ell, b)=\left(284^{\circ}, 75^{\circ}\right)\left(\mathrm{M} 87\right.$, top panel) and at $(\ell, b)=\left(320^{\circ},-30^{\circ}\right)$ (lower panel). The magnetic field is taken in the BSS-S configuration. The spectra correspond to the same sources as in Figure 6. 
Consider point sources at the same two particular directions chosen in Section 3 to illustrate the formation of multiple images: $(\ell, b)=\left(284^{\circ}, 75^{\circ}\right)$ (the Virgo cluster, visible from the northern terrestrial hemisphere) and $(\ell, b)=\left(320^{\circ},-30^{\circ}\right)$, visible from the southern terrestrial hemisphere. Both lie next to the super-galactic plane. At the highest energies, $E / Z$ larger than $100 \mathrm{EeV}$, the CRs will arrive to the Earth almost undeflected from the source direction and with negligible magnetic lensing effects (amplification $\simeq 1$ ). At lower energies the observed arrival directions change and multiple images appear, as described in Figure 6 in Section 3. In Figure 9 we follow the amplification factor of each image as a function of the energy, for the BSS$\mathrm{S}$ galactic magnetic field model. The top panel corresponds to the direction towards the Virgo cluster. The principal image is largely magnified around $E / Z=20 \mathrm{EeV}$, by a factor of order 5 . At around that same energy two new highly amplified images form. One becomes rapidly demagnified while the other remains magnified above the source. In the example in the southern sky (bottom panel) the principal image is steadily demagnified at energies below $30 \mathrm{EeV}$. Two highly magnified images form below 15 EeV but quickly become demagnified, another demagnified pair appears at energies below $6 \mathrm{EeV}$ and finally a new single and even more demagnified image is found at still lower energies. Clearly the spectrum observed at the Earth from each of the images would be the convolution of the injected spectrum with the plotted amplification.

\section{The Liouville theorem}

The Liouville theorem, i.e. the constancy of the phase space volume along the particle trajectories, has important implications for the effects we have been discussing here, making them essentially undetectable for energies well below the ankle. This theorem was first applied to CRs by Lemaitre and Vallarta [15] in their study of the deflection of low energy (few tenths of $\mathrm{GeV}$ ) CRs by the Earth dipole magnetic field. The conclusion [15, 16] was that, due to the Liouville theorem, an isotropic flux outside the solar system should remain isotropic when observed at the Earth (except for the Earth shadowing and the existence of blind regions corresponding to trajectories leaving the Earth which never manage to arrive to infinity [15] $]^{7}$. Since at low energies the CR propagation throughout the Galaxy is diffusive, the CR fluxes are essentially isotropic when arriving to the solar system and the theorem applies. In simple terms, when CR fluxes are magnified, the angular spread of their velocities increases (a well known problem for accelerator designers), so that they are seen as

\footnotetext{
${ }^{7}$ Something similar can happen to extra-galactic CRs, i.e. that when backtracking a CR out from the Earth it remains trapped in the galactic magnetic field. No extra-galactic sources will then be seen along these directions. This will however happen only for $E / Z \lesssim 1 \mathrm{EeV}$, and would imply that when projecting the "sky sheet" out from the Earth into the source plane, one patch will remain trapped inside the Galaxy, leaving a hole in the source sky.
} 
arriving from a larger solid angle. When measuring the flux per unit solid angle, the two effects compensate each other and an isotropic flux will remain isotropic. In gravitational lensing theory, this is also the reason why no anisotropies can be generated in the $\mathrm{CMB}$ radiation by lensing effects alone, which can only modify already existing anisotropies.

The magnification effects we have discussed before would rather have their gravitational analogue in the spectacular observation of strong lensing effects in quasars (multiple images) and also of microlensing observations in the Galaxy and the LMC (huge magnifications, caustic crossing due to binary lenses, etc.). Although with the present limited statistics the CR sky is not inconsistent with being isotropic, it is not expected to be so at the highest energies, where CR should arise from a few very violent sources (all with different intensities) and hence the Liouville theorem will not hide the effects analyzed above.

We note that, thanks to the Liouville theorem, there is an alternative way to compute the amplification for a given direction, besides the intuitive one explained before. It consists in following three nearby trajectories leaving the Earth with slightly different directions and the same energy. If the initial velocities are $\vec{v}_{0}$, $\vec{v}_{0}+\Delta \vec{v}_{i}(i=1,2)$, one may define a solid angle associated to those directions through $\mathrm{d} \Omega=\left(\Delta \vec{v}_{1} \times \Delta \vec{v}_{2}\right) \cdot \vec{v} /|\vec{v}|^{3}$. The magnification can then be equivalently computed as $\mathrm{d} \Omega_{E} / \mathrm{d} \Omega_{H}$, i.e. as the ratio of solid angles at the Earth and at the point where the particles leave the Galaxy $(r=20 \mathrm{kpc})$. We have verified that the two procedures agree in all directions to better than a percent for $E / Z>5 \mathrm{EeV}$, and this

gives us further confidence in the numerical accuracy achieved in the computation of the amplifications.

\section{Conclusions}

We have shown that the regular component of the galactic magnetic field acts as a giant lens for UHECRs. It can lead to multiple images, significant magnifications and deflections of the $\mathrm{CR}$ trajectories. These effects are important for $E / Z \lesssim 50 \mathrm{EeV}$, and hence can be relevant even for the highest energy events observed $(E \simeq 200$ $300 \mathrm{EeV})$ if the CRs have a component which is not light $(Z \geq$ a few). Below $E / Z \simeq 1 \mathrm{EeV}$, the trajectories become so tangled that this description is no longer useful, and also the galactic contribution to CR fluxes becomes dominant.

Actually, the same kind of magnetic lensing effect that we discussed here in detail for our Galaxy will also affect the CRs at their exit from the source galaxies. Hence, even if the deflections at the source galaxy will not significantly change the arrival direction to our galaxy (since the sources are far away), they can distort the original spectrum produced in the acceleration process.

We have emphasized the need to know the CR composition to do astronomy even at the highest energies. Moreover, a better knowledge of the galactic magnetic field is 
required. Besides establishing its general features, such as the existence of reversals in the spiral arms, the parity of the field across the galactic plane, the existence of an halo component and its precise scale height, also a better understanding of the magnetic field in the central parts of the Galaxy and whether a vertical component ( $B_{z}$ of a few tenths of $\mu \mathrm{G}$, induced e.g. by a galactic wind) actually exists would be important. In this sense, the results presented in this paper should be taken as indicative of the effects that are expected in realistic models, although the detailed predictions are model dependent.

Also the presence of a small scale random component could somehow affect the predictions, making the magnetic lens a not perfectly 'polished' one and then blurring the images. It is conceivable that with the large statistics expected with new detectors such as Auger [17], the identification of point sources and their study could also be helpful to understand the magnetic fields themselves.

The lensing effects we have discussed can have several implications. For instance, the study of the CR spectrum in a region around an observed source may show some of the features due to the magnification which were illustrated in Figure 9. These could be enhancements of the spectrum for energies leading to high magnification or when new images appear in a caustic. Also, some regions of the southern sky appear more demagnified at low energies (for the reference BSS-S model), while sources in the northern sky behave generally in the opposite way. This can lead to harder spectra observed in some regions of the southern sky and softer spectra in the north. This would be observable (with some care) if not too many sources dominate the sky, since otherwise sources from the north will be seen in the southern sky at low energies, softening the average spectrum seen in the south. It is also possible that the lensing effects could help in the observation of very faint sources, if they lie in regions of significant magnification.

\section{Acknowledgments}

Work partially supported by CONICET and Fundación Antorchas, Argentina.

\section{References}

[1] K. Greisen, Phys. Rev. Lett. 16 (1966) 748; G. T. Zatsepin and V. A. Kuzmin, Sov. Phys. JETP. Lett. 4 (1966) 78.

[2] J. L. Puget, F. W. Stecker and J. J. Bredekamp, Astrophys. J. 205 (1976) 638; L. N. Epele and E. Roulet, Phys. Rev. Lett. 81 (1999) 3295

[3] M. Giler, J. Wdowczyk and A. W. Wolfendale, J. Phys. G 6 (1980) 1561.

[4] G. Sigl, M. Lemoine and P. Biermann, Astropart. Phys. 10 (1999) 141. 
[5] T. Stanev, Astrophys. J. 479 (1997) 290.

[6] G. Medina Tanco, E. De Gouveia dal Pino and J. Horvath, Astrophys. J. 492 (1998) 200.

[7] N. M. Gerasimova and G. T. Zatsepin, Sov. Phys. JETP 11 (1960) 899; G. Medina Tanco and A. Watson, Astropart. Phys. 10 (1999) 157; L. N. Epele, S. Mollerach and E. Roulet, J. High Energy Phys. 03 (1999) 017.

[8] M. Takeda et al., "Small scale anisotropy of cosmic rays above $10^{19} \mathrm{eV}$ observed with the Akeno Giant Air Shower Array", astro-ph/9902239.

[9] K. O. Thielheim and W. Langhoff, J. Phys. A: Gen. Phys. 1 (1968) 694.

[10] R. Beck, A. Brandenburg, D. Moss, A. Shukurov and D. Sokoloff, ARA\&A, 34 (1996) 155.

[11] Y. Sofue and M. Fujimoto, Astrophys. J. 265 (1983) 722.

[12] S. Karakula, J. L. Osborne and W. Tkaczyk, J. Phys. A: Gen. Phys. 5 (1972) 904.

[13] A. A. Lee and R. W. Clay, J. Phys. G. 21 (1995) 1743.

[14] P. Schneider, J. Ehlers and E. E. Falco, Gravitational Lenses, Springer-Verlag, 1992.

[15] G. Lemaitre and M. S. Vallarta, Phys. Rev. 43 (1933) 87.

[16] W. F. G. Swann, Phys. Rev. 44 (1933) 224.

[17] http://www.auger.org/auger.htm] 
